\title{
El papel de Raúl Prebisch en la creación del Banco Central de la República Argentina
}

\author{
Florencia Sember
}

Resumen. El objetivo de este trabajo es estudiar el papel de Prebisch en la creación del Banco Central de la República Argentina. La concepción de Prebisch sobre las funciones e instrumentos del banco central deriva de su explicación del ciclo económico argentino. Se muestra que las modificaciones introducidas por el economista argentino al proyecto británico de 1933 reflejan dos concepciones diferentes en lo relativo a las cuestiones monetarias y bancarias. El proyecto británico, escrito por Otto Niemeyer, está impregnado por la treasury view: demostraba temor a la participación estatal y a la inflación y limitaba al mínimo el margen de discrecionalidad de las autoridades monetarias. Las modificaciones introducidas por Prebisch reflejan un conocimiento más acabado del funcionamiento del ciclo económico argentino, otorgando al banco central una multiplicidad de instrumentos no contemplados por Niemeyer $y$ garantizando una mayor libertad a las autoridades monetarias para aplicar medidas discrecionales de acuerdo a la coyuntura económica.

Palabras clave: Raúl Prebisch, Otto Niemeyer, Banco Central de la República Argentina, banca central, Argentina.

Abstract. This article seeks to analyze Prebisch's role in the creation of the Central Bank of Argentina. Prebisch derived his ideas regarding the functions and instruments of the central bank from his explanation of the Argentinian economic cycle. It is demonstrated that the modifications introduced by the Argentinian economist to the British project of 1933 reflect two different conceptions of monetary and banking issues. The British project, written by Otto Niemeyer, is imbued with the treasury view: it reflects fears of government participation and inflation, and it restricts the discretionary power of monetary authorities to a minimum. The modifications introduced by Prebisch reflect a more complete knowledge of the functioning of the Argentinian economic cycle, providing the central bank with multiple instruments not considered by Niemeyer and granting monetary authorities more freedom to apply discretionary measures according to the prevailing economic situation.

KeYwords: Raúl Prebisch, Otto Niemeyer, Central Bank of Argentina, central banking, Argentina.

Florencia Sember es economista e historiadora argentina. Becaria posdoctoral del Consejo de Investigaciones Científicas y Técnicas (CONicet). 


\section{INTRODUCCIÓN}

It is Argentina which affords the most striking example of cycle neutralisation in both boom and recession during the three years 1936-38 [...] The changes in the world market affected the Argentine balance of payments with a particular violence $[. .$.$] The repercussions of these$ events on the domestic credit situation were surprisingly small, owing to the remarkable policy of monetary "insulation" adopted (Ragnar Nurkse, International Currency Experience, 1944)



aúl Prebisch es, en general, recordado por su actuación en la Comisión Económica para América Latina y el Caribe (CEPAL), como el generador y promotor de una visión autóctona de los problemas económicos latinoamericanos. De acuerdo a esta visión, el punto de partida del pensamiento de Prebisch sería su famoso texto «El desarrollo económico de la América Latina y algunos de sus principales problemas» (Prebisch, 1949), considerado el «manifiesto de la CEPAL» (Albert Hirschman, 1961). Sin embargo, a pesar de haber sido una personalidad muy activa desde 1949, Prebisch pasó la mitad de su vida en Argentina, donde ocupó cargos importantes en diversas instituciones y tuvo un rol preponderante en la creación del Banco Central.

En su libro La fantasía organizada, Celso Furtado afirma que en 1950, cuando Prebisch llegó a Santiago de Chile para incorporarse a la CEPAL, era el único economista latinoamericano de renombre (Furtado, 1988:51). ¿Por qué era reconocido Prebisch en esos años? El economista argentino había sido ge- 
rente general del Banco Central de la República Argentina (BCRA) entre 1935 y 1943, y había sido asesor sobre cuestiones monetarias y bancarias en otros países latinoamericanos. Prebisch había participado activamente en la misión de Robert Triffin en Paraguay y había sido invitado a Venezuela, República Dominicana y México como asesor sobre esas mismas cuestiones. Las políticas anticíclicas llevadas adelante por el BCRA, bajo la guía de Prebisch, fueron vistas como un ejemplo para las economías exportadoras de bienes primarios. ${ }^{2}$ Ragnar Nurkse y Robert Triffin fueron entre los pocos economistas que reconocieron el legado de Prebisch en cuestiones relativas a la banca central. ${ }^{3}$

El objetivo de este trabajo es estudiar el papel de Prebisch en la creación del BCRA. El economista argentino no era un teórico de la banca central: su contribución aparece desde el inicio como pragmática, basada en sus conocimientos sobre el funcionamiento de la economía argentina. Esto no significa que Prebisch no haya tenido una concepción de banco central, sino que en lugar de abordar el problema, teniendo en mente ciertos principios teóricos, lo veía desde el punto de vista de los objetivos, funciones e instrumentos del banco. Prebisch vio en la creación del BCRA una oportunidad para resolver los problemas que había identificado estudiando la economía argentina en la década de los veinte. En consecuencia, su concepción de las funciones del banco central deriva de su explicación del ciclo económico argentino.

\footnotetext{
/1/ Sobre esto, ver Helleiner (2009).

12/ La política anticíclica evitaba la deflación en las fases descendentes del ciclo, pero también contenía la expansión en las fases ascendentes, lo que no permitió al país crecer durante el período como lo hicieron otros países en situación similar. O'Connell (1984) muestra que la participación del sector manufacturero en el PBI aumentó solamente de $18 \%$ en 1929 a $20.9 \%$ en 1939 , y que este crecimiento industrial fue intensivo en mano de obra y estuvo basado en el uso intensivo de plantas ya existentes.

13/ Triffin decía que Prebisch fue uno de los mejores economistas que conoció (ver Pazos, 1988: 190). Ver también Triffin (1966: 141, nota 2) y Helleiner (2009). Sayers (1976: 524) reconocía que Prebisch era «a man destined for high place in the annals of central banking», pero no reconoció el importante papel que éste tuvo en la creación del BCRA.
} 
En la segunda sección se estudiarán las condiciones institucionales y políticas que precedieron a la creación del Banco Central de la República Argentina. En la tercera sección se analizará el rol de Prebisch en el diseño final del nuevo sistema monetario y bancario, poniendo énfasis en las modificaciones que realizó al proyecto propuesto por el experto británico Otto Niemeyer. Finalmente, en la última sección se incluyen algunos comentaros finales a modo de conclusión.

\section{LA NECESIDAD DE UN BANCO CENTRAL}

En la primera mitad de la década de los treinta hubo diferentes propuestas para establecer un banco central en Argentina, que fue finalmente creado en 1935. El primero de estos proyectos fue elaborado en $1931^{4}$ por una comisión creada por Enrique Uriburu, el ministro de Finanzas del gobierno militar que había comenzado en 1930. Hubo principalmente dos factores que influyeron en esta decisión. Por un lado, estaban las condiciones económicas e institucionales de la época: diferentes instituciones desempeñando funciones que se superponían y carecían de coordinación y una importante crisis mundial. Por otro lado, había una fuerte presión británica en este sentido desde 1929, año en que Argentina abandonó el patrón oro. ${ }^{5}$ Estos dos factores determinaron la formación de la Comisión Uriburu, en la cual Prebisch participó activamente.

En cuanto a las condiciones institucionales, Argentina contaba con tres instituciones que desempeñaban funciones que luego corresponderían

14/ Éste no fue el primer proyecto en la historia argentina, sino el primero de la serie de proyectos de inicios de los años treinta que culminaron con la creación del banco en 1935.

15/ Argentina y Gran Bretaña habían desarrollado una relación comercial muy cercana: Gran Bretaña absorbía una parte importante de las exportaciones argentinas y casi todas las exportaciones de carne, y poseía importantes inversiones en Argentina, las más importantes de las cuales eran las compañías ferroviarias. 
al banco central (Prebisch, 1934: 63-65). En primer lugar estaba la Caja de Conversión, creada en 1890 con el propósito de convertir gradualmente los billetes emitidos durante la desastrosa experiencia de la Ley de Bancos Garantidos de $1887 .{ }^{6} \mathrm{La}$ Caja de Conversión era la única institución autorizada a emitir billetes y debía acumular un Fondo de Conversión con el objetivo de implementar la convertibilidad en el futuro. Sin embargo, la convertibilidad estaba subordinada a los flujos de capitales externos y, por lo tanto, funcionó intermitentemente, primero en 1903-1914 y nuevamente en 1927-1929. La diferencia entre esta institución y una caja de conversión tradicional era que ésta no estaba obligada a convertir todo el oro que ingresaba al país en papel moneda, con lo cual el oro podía también destinarse a atesoramiento privado. Asimismo, no todas las exportaciones de oro provenían necesariamente de la Caja de Conversión, sino que podían provenir de individuos privados o de las reservas de los bancos (ver Ford, 1962: 96).

Hacia los treinta, el rol de la Caja de Conversión era muy distinto al planeado. En 1931 una ley de redescuento la autorizó a redescontar documentos del Banco de la Nación Argentina. ${ }^{7}$ La ley de redescuento había sido pensada justamente para evitar una contracción monetaria, aunque este instrumento:

No debe ser usado, en modo alguno, para estimular nuevos negocios o ampliar los existentes, o para facilitar inversiones a largo plazo que, por muy útiles que fuesen, deben ser postergadas a épocas más holgadas del mercado monetario, sino para permitir el desenvolvimiento regular del crédito, dando a los bancos el medio de afrontar sus exigibilidades inmediatas sin necesidad de acudir a la brusca contracción de los préstamos (Prebisch, 1931:2).

16/ Esta ley fue un intento de recrear el Sistema de Bancos Nacionales de Estados Unidos. Cualquier banco que cumpliese determinadas condiciones estaba autorizado a emitir.

17/ El Banco de la Nación Argentina (BNA) era el banco público más importante, creado después de la crisis Baring de 1890, y ejercitaba algunas funciones que corresponderían a un banco central. 
Un año más tarde, la Caja de Conversión fue autorizada a emitir billetes contra los títulos del «Empréstito patriótico» de 1932. El resultado fue que hacia 1932, las funciones de la Caja de Conversión eran mucho más amplias de lo planeado inicialmente y habrían correspondido a un banco central.

Algunos meses después de la Ley de Redescuentos, se implementó el control de cambios, con el fin de intentar llegar a un equilibrio en la balanza de pagos mediante un control selectivo de las importaciones. El decreto que imponía el control de cambios fue redactado por el mismo Prebisch (Magariños, 1991: 68). Luego de que Gran Bretaña abandonara el patrón oro en 1931, se esperaba una ulterior devaluación del peso moneda nacional. Hacia diciembre de 1931, los intentos de sostener el tipo de cambio habían costado m $\$ \mathrm{n} .389$ millones (Prebisch, 1944: 13). La única salida era el control de cambios.

Aún si había sido implementado como una medida de emergencia, Prebisch se convenció con el tiempo de que el control de cambios debía ser un complemento a la política monetaria del banco central, al que podría recurrir o no de acuerdo a las condiciones económicas prevalecientes. Era visto como:

Un instrumento valioso que nos permita establecer en un país de la fisonomía económica de la Argentina, un compromiso razonable entre el sistema rígido del patrón oro, que hemos visto que sometía a la economía del país a grandes e intensas fluctuaciones, y el régimen de moneda inconvertible, depreciada y fluctuante, que tiene también sus graves inconvenientes (Prebisch, 1944: 10).

Por último, estaba el Banco de la Nación Argentina (BNA), que también desempañaba algunas funciones que corresponderían a un banco central: administraba las cuentas del gobierno, hacía redescuentos a los otros bancos ${ }^{8} y$

18/ Después de la Ley de Redescuentos, estos documentos a su vez podían ser redescontados en la Caja de Conversión. 
manejaba la Cámara Compensadora. Este banco había sido creado luego de la quiebra del Banco Nacional durante la crisis Baring de $1890 \mathrm{y}$, dado que en el momento de su creación no se habían encontrado suscriptores para sus acciones, era público.

Durante su desempeño como director de la Oficina de Investigaciones Económicas del BNA, ${ }^{9}$ Prebisch había notado que este banco no se comportaba como el resto. La especificidad de este banco puede verse en las estadísticas publicadas en la Revista Económica. El BNA hacía algunos intentos por neutralizar las fluctuaciones y por eso su coeficiente de reservas muestra fluctuaciones mucho mayores que en el resto de los bancos (figura 1). Esto sucedía a causa de la doble naturaleza del BNA, que además de ser un banco comercial se comportaba como cuasi banco central.

\section{FIGURA I}

Encajes del BNA y los otros bancos (1926-1934)

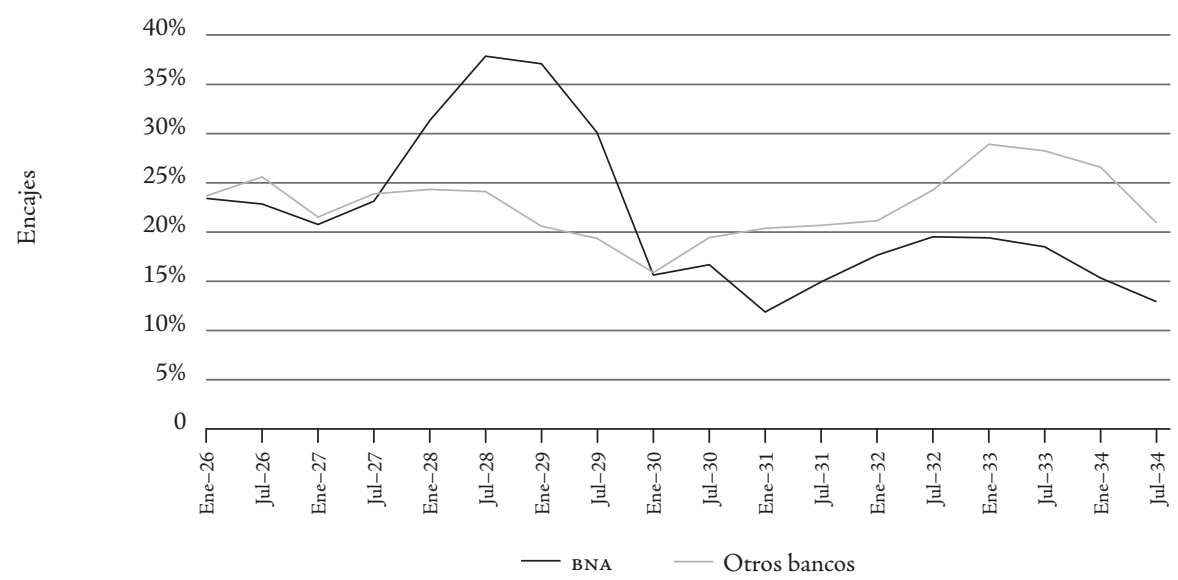

Fuente: Revista Económica del BNA, núm. 1 de 1928 hasta el núm. 9-12 de 1934.

19/ Prebisch fue nombrado director de esta oficina y director de su revista, la Revista Económica del BNA, en 1928. 
Cuando comenzó el éxodo de capitales a mediados de 1928, los otros bancos pudieron mantener sus reservas transfiriendo el problema al BNA. Esto podía hacerse de forma directa, es decir, reduciendo los depósitos en dicho banco o descontando documentos, o indirectamente reduciendo los depósitos en la Cámara Compensadora. La variación de las reservas del BNA era el único elemento de flexibilidad en el sistema, que evitaba grandes fluctuaciones en la cantidad de circulante. Pero estas variaciones atentaban contra las actividades comerciales del banco. Como afirma Goodhart:

In order to be effective in controlling banking cycles, it is necessary for a Central Bank to supplement support action at times of crises with restrictive measures during periods of general optimism and expansion. The latter is extremely difficult, probably impossible, for a bank competitively seeking to maximize profits (Goodhart, 1988: 54).

Prebisch estaba de acuerdo con esta afirmación, pero en el caso del BNA su mayor preocupación era que, si continuaba siendo público después de la creación del banco central, podría haber conflictos entre los dos bancos. El gobierno podría presionar al BNA para obtener préstamos al mismo tiempo que el banco central intentaba llevar adelante una política restrictiva, socavando así sus efectos. ${ }^{10}$

Además de las características institucionales, que hacían necesaria la creación de un banco central, el segundo factor importante fue la presión británica en este sentido. Argentina tenía una intensa relación comercial con Gran Bretaña, de la cual dependían los sectores políticamente más influyentes del país. A su vez, el capital inglés invertido en el país era muy importante en el sector de

/10/ Prebisch tenía la idea de convertirlo en banco mixto luego de la creación del BCRA, cosa que finalmente no se hizo. Cuando se decidió crear el banco central como institución separada del BNA, este banco se opuso con fuerza. 
los ferrocarriles, los servicios públicos y los préstamos públicos. El abandono del patrón oro por parte de Argentina en 1929 había generado incertidumbre en los inversores británicos en cuanto a la posibilidad de repatriar las libras esterlinas provenientes de los beneficios de sus inversiones. Estos temores se acentuaron cuando en 1931 Argentina implementó el control de cambios.

A raíz de esta situación, a partir de 1929 los británicos comenzaron a discutir la necesidad de que Argentina estableciese un banco central ortodoxo, adoptando un patrón de cambio oro basado en la libra esterlina. Así, el país constituiría sus reservas en libras esterlinas y establecería un tipo de cambio fijo con la libra, lo que garantizaría a las empresas británicas en Argentina la estabilidad de sus beneficios en libras. Luego del golpe de Estado de 1930, en Argentina se aplicó un fuerte ajuste fiscal, que entusiasmó a los británicos porque tenían la visión de que el equilibrio fiscal era una condición necesaria para mantener estable el tipo de cambio. A partir de ese año, el Banco de Inglaterra inicia gestiones para que una misión británica fuese invitada por Argentina (Sember, 2010: Sección 3.2).

En 1932, el ministro de Finanzas del nuevo gobierno de Agustín P. Justo, Alberto Hueyo, decidió solicitar asesoramiento al Banco de Inglaterra, oficialmente con el objetivo de estudiar y proponer una reforma del sistema monetario y bancario. En realidad, el gobierno esperaba que la invitación la diese una especie de sello de confianza ante el parlamento donde tenía dificultades para aprobar el presupuesto. Asimismo, la misión debía aconsejar al gobierno en materia fiscal, aunque ese aspecto no fue hecho público. ${ }^{11}$ Esta invitación era en realidad esperada por los británicos desde 1930. El líder de la misión británica, que llegó a principios de 1933, era sir Otto Niemeyer, un representante emblemático de la treasury view. El proyecto propuesto por Niemeyer en 1933 no se implementó, en parte por la oposición del BNA, y en 1934 Federico

111/ De hecho, uno de los integrantes de la misión británica era Norman Young, un funcionario del tesoro. 
Pinedo le encomendó a Prebisch la tarea de revisarlo. Los significativos cambios introducidos por Prebisch son los que hicieron posible las políticas anticíclicas de la segunda mitad de la década de 1930. El proyecto fue aprobado por el Congreso finalmente a inicios 1935, con muy pocos cambios respecto al presentado por Prebisch. El Banco Central de la República Argentina (BCRA) comenzó a funcionar en mayo de 1935.

\section{LA CONCEPCIÓN DEL BANCO CENTRAL}

Prebisch sostenía que en la reorganización del sistema monetario y bancario había que tener en cuenta dos aspectos:

a) Las características fundamentales de Argentina como país agroexportador, altamente dependiente de las fluctuaciones de los precios y cantidades de las exportaciones y extremamente vulnerable a los flujos de capital extranjero. Era esencial tener en cuenta la vulnerabilidad externa en el diseño del nuevo sistema.

b) La delicada situación bancaria producto de la crisis. Un sistema que comenzase a funcionar sin tener en cuenta este aspecto sería defectuoso desde el inicio.

Para contemplar el primer punto, Prebisch pensará en un banco central que contase con todos los instrumentos necesarios para llevar adelante políticas anticíclicas. Para tener en cuenta el segundo punto, además de un banco central encargado de la supervisión del sector bancario y una ley bancaria, se crea el Instituto Movilizador de Inversiones Bancarias, ${ }^{12}$ que tenía la función de adquirir las inversiones congeladas de los bancos y liquidarlas gradualmente.

/12/ Esta institución estaba contemplada en el proyecto Uriburu de 1931, pero ausente en el proyecto británico. 
Prebisch había estudiado detenidamente el funcionamiento del ciclo económico argentino ${ }^{13}$ durante la década de 1920, intentado explicar lo que más tarde denominó "patrón oro esporádico». ${ }^{14}$ Los flujos de capitales eran exógenos, pues dependían de las condiciones reinantes en los mercados financieros internacionales. Sobre este punto, Prebisch citaba a Tugan Baranowsky (1913), que había mostrado que los flujos de capital desde los países industriales hacia los países periféricos dependían principalmente de las oportunidades de inversión en Europa (Prebisch, 1921-1922: 125, nota 66). Cuando estas oportunidades no eran suficientemente atractivas, los capitales buscaban mayores rendimientos en los países periféricos. El ingreso de capitales permitía el retorno a la convertibilidad, pero también, mediante el aumento de reservas de los bancos, generaba una expansión monetaria excesiva. ${ }^{15}$ Así comenzaba la fase ascendente del ciclo económico, caracterizada por el ingreso de capitales, expansión de la moneda y el crédito e importaciones ${ }^{16}$ cada vez mayores. El progresivo aumento de las importaciones y la acumulación de servicios financieros hacían necesarios ingresos de capital más elevados. Cuando estos capitales dejaban de ingresar al país, muchas veces por razones exógenas, ${ }^{17}$ se producía una crisis de balanza de pagos, se abandonaba la convertibilidad y comenzaba la fase descendente del ciclo.

113/ Para un examen detallado de la explicación de Prebisch del ciclo económico, ver Pérez Caldentey y Venrengo (2012) y Sember (2010; capítulo 2).

/14/ Prebisch llamaba al sistema monetario argentino «patrón oro esporádico» (Prebisch, 1932: 43), porque mostraba a lo largo de su historia recurrentes periodos de patrón oro alternados con periodos de inconvertibilidad. El patrón oro funcionó en 1866-1876, 1883-1885 (en este caso era bimetálico), $1899-1914$ y $1927-1929$.

115/ Esto se veía acentuado, según Prebisch, por el comportamiento irresponsable de muchos bancos en tiempos de prosperidad.

116/ Prebisch había notado que la elasticidad ingreso de las importaciones era alta, con lo cual éstas aumentaban excesivamente durante los periodos de crecimiento económico.

/17/ Estos fueron, por ejemplo, los casos de 1914, por el inicio de la Primera Guerra Mundial, y 1928, por el boom en Wall Street. 
De acuerdo con esta interpretación del ciclo económico, para Prebisch iba a ser fundamental que el banco central estuviese dotado de todos los instrumentos necesarios para controlar los mecanismos que actuaban en las fases ascendentes y descendentes del ciclo. La relación entre los flujos externos de capital y la cantidad de circulante será manejada a través de la esterilización y el redescuento. La relación entre la emisión y el crédito estará subordinada al respeto por los bancos de las nuevas regulaciones bancarias, que el banco central debía hacer respetar. El nexo entre la actividad interna y la balanza de pagos sería mediado por el sistema de control de cambios.

\section{Los instrumentos del banco central}

De acuerdo con esta concepción, el banco central debía perseguir una política monetaria activa, aumentando o restringiendo el crédito de acuerdo a la situación económica. Los instrumentos para lograr esto eran el redescuento, los adelantos, las operaciones de mercado abierto y las operaciones con oro y moneda extranjera.

En el proyecto de Niemeyer, el manejo de la tasa de redescuento era el único instrumento otorgado al banco central para regular la liquidez del sistema. Prebisch consideraba que el instrumento más eficiente para esto eran las operaciones de mercado abierto, ${ }^{18}$ porque en caso de que fuese necesaria una restricción del circulante en un momento en que los bancos no necesitaban redescontar, el aumento de la tasa de redescuento no produciría ningún resultado. Estas operaciones no estaban contempladas en el proyecto británico, pero Prebisch se había informado sobre su funcionamiento en su visita al Banco de Inglaterra en 1933. Niemeyer se había opuesto activamente a la inclusión de

/18/ Este será efectivamente el principal instrumento utilizado por el BCRA durante el periodo en que Prebisch fue gerente general. 
este instrumento en la carta orgánica del banco y en 1933 intentó convencer a Prebisch de su inconveniencia: «Prebisch is still here and came to see me the other day. I hope shortly to carry him off to the country for a couple of days, in which period I will endeavour to inoculate him with the true virus» (Niemeyer to Meynell, 26 ${ }^{\text {th }}$ may, 1933).

El proyecto británico apuntaba a dejar al banco el menor margen de discrecionalidad posible. Esto también era evidente en lo relativo a la garantía metálica de los billetes. Si bien ambos proyectos contemplaban una garantía metálica por los billetes y depósitos, el proyecto británico imponía al banco medidas correctoras en el caso de que la garantía metálica cayese por debajo de 33\%. El banco tenía la obligación de aumentar su tasa de descuento ante una disminución importante de las reservas, y este aumento debía ser mayor cuanto más lejos estuviesen las reservas de 33\%. Prebisch dejaba en claro que no proponía normas estrictas para hacer cumplir este límite, que en su caso era de $25 \%$, con el fin de dar al BCRA suficiente margen de maniobra para manejar situaciones críticas. Consideraba que si se había llegado a esa situación, seguramente la coyuntura económica era delicada y subir la tasa de redescuento podría agravarla aún más (por ejemplo, forzando la liquidación en un año de malas cosechas). La única restricción impuesta era que en ese caso el banco no podía distribuir utilidades. Esto para Niemeyer reflejaba que Prebisch había caído en el error, muy común después de la Gran Depresión, de tener temores infundados a la deflación.

Asimismo, se advierte en Niemeyer una intención de dejar el menor margen posible a la discrecionalidad de las autoridades del banco. Según la concepción de Prebisch, para garantizar el buen funcionamiento de la institución bastaba con que fuese independiente del gobierno, dejando a las autoridades autonomía para decidir los pasos a seguir según la situación económica del momento. Niemeyer, por el contrario, proponía reglamentaciones mucho más rígidas, que indicaban los pasos a seguir en cada caso. 


\section{Convertibilidad y politica cambiaria}

En cuanto al patrón oro y la política cambiaria, Prebisch, al contrario de Niemeyer, no daba por sentado que el mundo retornaría en el futuro al patrón oro. $\mathrm{Su}$ proyecto daba lugar a otras posibilidades.

Si el mundo vuelve con prontitud a lo que se ha considerado como normalidad monetaria y si las principales monedas hoy inconvertibles retornan al patrón oro, estas reformas habrán preparado el terreno para que la moneda argentina recupere también su estabilidad sobre bases más adecuadas al carácter peculiar de nuestra economía. Pero si en vez de volverse al patrón oro generalizado y a una relativa libertad en las transacciones internacionales, el mundo continuara presentando el cuadro de economías cerradas, con monedas autónomas, la República, gracias a una nueva organización de la moneda y el crédito, podrá proseguir su propia política cuidando sus propios intereses y evitando en lo posible que esas circunstancias deterioren sus fundamentales instituciones económicas (Prebisch, 1934: 62).

El proyecto presentado por Prebisch otorga al banco central la posibilidad de implementar el control de cambios en caso de que no se retornase al patrón oro. Por el contrario, en su informe Niemeyer sostenía que:

Un sistema de Banco Central evidentemente no puede producir todos sus efectos, si no existe $-y$ mientras no exista - un cambio exterior libre, que se funde sobre una paridad legal de la moneda nacional, hacia cuyo logro deben dirigirse todos los esfuerzos (Niemeyer, 1933: 133).

Cuando Niemeyer hablaba de paridad legal de la moneda nacional, pensaba en una paridad con la libra esterlina. De hecho, en su proyecto, las 
reservas podían constituirse indistintamente en oro en divisas convertibles. Prebisch, por el contrario, no era favorable a mantener las reservas en divisas, que limitó a un máximo de $10 \%$, porque había sido testigo de las pérdidas sufridas durante la Gran Depresión por los bancos que mantenían reservas en divisas de países que luego abandonaron la convertibilidad. La crisis había demostrado que aún las divisas más fuertes podían abandonar el patrón oro.

El proyecto británico era funcional al fortalecimiento del sterling bloc, que era considerado una prioridad por Montagu Norman, el presidente del Banco de Inglaterra (Sayers, 1976: 513). Asimismo, las empresas británicas en Argentina ejercían presiones en el mismo sentido, ya que con un tipo de cambio fijo con la libra no tendrían problema en repatriar sus utilidades. Incluso luego de que el proyecto modificado por Prebisch estuviese terminado, por el lado británico se seguía insistiendo en incorporar a Argentina al bloque de la libra:

We wonder whether as an alternative to the Minister of Finance's proposal for the revaluation of gold, it would be possible to put up some scheme whereby the peso was based on sterling and would follow the upward or downward course of the pound, to be stabilized in the future when the pound is stabilized (Meynell to Peacock, $10^{\text {th }}$ december, 1934).

Revaluación del oro y su uso

El proyecto presentado por Prebisch en 1934, al igual que el de 1931, estaba basado en la revaluación del oro en la Caja de Conversión, que estaba valuado al tipo de cambio de la Ley de Convertibilidad de $1899 .{ }^{19}$ El precio de mercado del oro era mucho más alto del precio al que estaba valuado en la caja y Prebisch no pensaba que había posibilidades de que bajase en un futuro

${ }^{/ 19 /}$ Este tipo de cambio era de $\mathrm{m} \$ \mathrm{n} 2,27$ por peso oro. 
próximo. Por lo tanto, el oro debía revaluarse en el momento de la creación del вCRA, asegurando que los beneficios de esa revaluación no fuesen empleados de forma inflacionaria, es decir, para aumentar la cantidad de circulante. Para Prebisch, la cuestión del uso de los beneficios de la revaluación era también importante en otro sentido: la Caja de Conversión era un ente público y también lo era el oro en su posesión. Si este oro iba a pasar a una institución mixta, como era el BCRA, se debía al menos garantizar que los beneficios de la revaluación fuesen al Estado nacional. Por eso, los beneficios de revaluación van a acreditarse al gobierno nacional en una cuenta en el BCRA:

\section{FIGURA 2}

La creación del BCRA a partir de la Caja de Conversión.

\begin{tabular}{|c|c|c|c|c|}
\hline \multicolumn{2}{|c|}{$\begin{array}{l}\text { CAJA DE CONVERSIÓN } \\
\text { (MILLONES DE M\$N) }\end{array}$} & & \multicolumn{2}{|c|}{$\begin{array}{c}\text { Banco Central } \\
\text { (MILLONES DE M\$N) }\end{array}$} \\
\hline Activo & Pasivo & & Activo & Pasivo \\
\hline $\begin{array}{l}\text { Oro.. } 561 \\
\text { Otros... } 650\end{array}$ & Emisión... 1211 & & $\underset{\text { Otros... } 650}{\text { Oro...1061 }}$ & $\begin{array}{c}\text { Emisión... } 1211 \\
\text { Depósitos del gob. nacional... } 500\end{array}$ \\
\hline 1211 & 1211 & & 1711 & 1711 \\
\hline
\end{tabular}

La figura 2 muestra el balance de la Caja de Conversión, a partir del cual se va a constituir el BCRA, y la revaluación del oro, que es acreditada en una cuenta al gobierno nacional. ${ }^{20}$ Estos beneficios no podían ser utilizados arbitrariamente, sino que debían der utilizados de la siguiente manera:

+ Para cancelar deudas con el Banco Nación, que tenía serios problemas de liquidez (m\$n 200 millones).

/20/ Estas son las cifras tentativas expuestas por Prebisch en el proyecto, las cifras reales son diferentes pero el mecanismo no cambia. 
+ Para incrementar la liquidez de otros bancos, a través del Instituto Movilizador de Inversiones Bancarias (гмів) ( $\$$ \$n 68 millones).

+ Para comprar documentos redescontados por los bancos en la Caja de Coversión, también a través del ıмів ( $\mathrm{m} \$ \mathrm{n} 212$ millones).

- Para capitalizar al ıмıв у al всRA (m\$n 10 millones a cada institución).

El Instituto Movilizador de Inversiones Bancarias debía permitir al BCRA comenzar sus funciones libre de los obstáculos dejados atrás por la crisis. El instituto tenía como función comprar los activos congelados de los bancos e intentar liquidarlos gradualmente para obtener el mayor rendimiento posible.

Niemeyer no había pensado en ninguna solución al problema de la insolvencia de los bancos dejado atrás por la crisis. Veía con desconfianza la creación de dicha institución por dos motivos: el primero era la participación del gobierno, a la cual era contrario por considerar que todo lo relativo al sistema de banco central debía ser completamente independiente. De hecho, su proyecto contemplaba un banco central con capital menor que el de Prebisch y cuyas acciones no podían ser suscriptas por el Estado nacional. ${ }^{21}$ En segundo lugar, temía que esta institución generase inflación:

Además, sin duda Ud. apreciará que contiene un peligro potencial considerable de inflación el proyecto de Banco Movilizador, a menos que no se tome mucho cuidado. No puedo creer que haga falta un aumento en la circulación en la Argentina, sea en billetes o en medio circulante creado por el Banco Central (Niemeyer, 1935: 106)

Niemeyer no estaba de acuerdo con la revaluación del oro, que era la base del proyecto de Prebisch. Sin embrago, no había pensado en ninguna so-

${ }^{/ 21 /}$ En el proyecto final, el $50 \%$ de las acciones serán suscriptas por el Estado nacional. 
lución alternativa para el sistema bancario. Según Prebisch, en la Conferencia Económica de Londres, le preguntó a Niemeyer:

«Sir Otto, ¿̇cómo ha considerado usted el problema, que a mí me parece muy grave en la Argentina, de la cartera congelada del sistema bancario?» «Ah - dijo-, con un alza de precios internacionales del 10 ó 15\% todo esto se corrige». Habían llamado al célebre médico sin abrirse, sin dejar ver el cuerpo, por pudor. No tenía una idea... (Magariños, 1991: 112).

Sin embargo, en el Banco de Inglaterra circulaban informes internos sobre la situación bancaria en Argentina, por lo cual es difícil pensar que Niemeyer ignorara su gravedad. Probablemente pensaba que lo mejor era dejar quebrar algunos bancos, ya que consideraba que en Argentina había demasiados bancos en proporción al nivel de actividad económica (Sember, 2010: Sección 3.2).

Si bien los británicos no confiaban en la participación estatal en el пмів y aseguraban que la revaluación del oro sería inflacionaria, debieron reconocer que Niemeyer no había pensado en ninguna solución a la insolvencia del sector bancario.

\section{La política bancaria}

Como mencionamos en la introducción, no sólo era importante la creación de un banco central, sino que era esencial para su buen funcionamiento un sistema bancario regulado por una ley bancaria y supervisado por el banco central.

Según Prebisch, los bancos desempeñaban una función que implicaba responsabilidad pública, no sólo porque mantenían los ahorros de los clientes, sino porque podían incidir en la situación monetaria a través del crédito. La diferencia con otro tipo de empresas comerciales era evidente, sobre todo en tiempos de crisis, porque: 
Mientras las consecuencias de la caída de una empresa comercial cualquiera, sólo alcanzan generalmente a las que le son conexas, la de un banco puede propagar rápidamente la alarma de sus depositantes a los de otros bancos, y engendrar así un pánico que afecte tanto a los buenos establecimientos como a los que no se han conducido con la necesaria cautela (Prebisch, 1934: 83).

La ley bancaria establecía reglas que debían respetar los bancos, mecanismos de supervisión y contemplaba sanciones. En general, la aplicación de la ley correspondía al BCRA, excepto algunas tareas que eran dejadas a otras agencias gubernamentales. Por ejemplo, para el establecimiento de un nuevo banco, el BCRA debía emitir su opinión, pero la decisión le correspondía al presidente de la república, que se suponía que representaba mejor los intereses de la comunidad. Lo mismo sucedía con las sanciones. Correspondía al BCRA controlar si los bancos respetaban la ley, pero no imponer sanciones.

Contrariamente a Niemeyer, Prebisch incluía la supervisión del sistema bancario en el banco central por varias razones:

+ Para verificar la calidad de los documentos redescontados, era necesario conocer el estado de los bancos que solicitaban ese redescuento, por eso el banco central debía tener acceso a la información sobre los bancos.

+ Porque facilitaba al BCRA la comprensión del sistema económico en el cual operaba.

+ Evitaba una burocracia excesiva. Si se delegaba esta tarea a un organismo diferente, podría pasar que éste no recolectase toda la información necesaria, que debería ser luego recolectada por el banco central, duplicando así las tareas y las jurisdicciones.

Otra diferencia interesante con el proyecto de Niemeyer, es que Prebisch eliminó el requisito para los nuevos bancos de un capital mayor a $\mathrm{m} \$ \mathrm{n}$. 
1’000,000. Prebisch justificó esta decisión porque de esta manera se permitía funcionar a pequeños bancos cooperativos o privados, especialmente de áreas rurales: "No es en sí misma la magnitud del capital, sino la forma en que opera, lo que decide si un establecimiento de crédito reporta o no un servicio positivo para la colectividad» (Prebisch, 1934:84).

El encaje mínimo establecido por la ley de bancos era menor que el propuesto por el proyecto Niemeyer: $16 \%$ para depósitos a la vista y $8 \%$ para los demás depósitos. La intención de Prebisch era evitar dar disposiciones muy meticulosas al respecto para asegurar la liquidez del sistema, dejando al BCRA la posibilidad de autorizar transitoriamente que estos límites no se respetasen. En un país agrícola como era Argentina, sin mecanismos para financiar a la industria, normas muy rígidas hubiesen sido imposibles de respetar. Nuevamente, la cuestión de la discrecionalidad aparece en el proyecto de Prebisch, en contraste a la propuesta británica.

\section{Comentarios finales}

En este trabajo se puso en evidencia el importante papel de Raúl Prebisch en la creación del Banco Central de la República Argentina. Si bien el proyecto de banco central presentado al Congreso en enero de 1935 es considerado muchas veces como producto de la concepción británica sobre la banca central, se mostró que Prebisch le realizó modificaciones sustanciales, sin las cuales el banco no hubiese tenido los instrumentos necesarios para aplicar políticas anticíclicas.

El proyecto británico era un reflejo de lo que en esos años se consideraba un banco central ortodoxo. Los fuertes temores a la inflación y a la intervención estatal eran elementos recurrentes en el proyecto presentado por Niemeyer, que es considerado uno de los más emblemáticos representantes de la treasury view. El interés en el papel de la libra en un periodo en el cual todos los países estaban abandonando el patrón oro y los intereses de las empresas británicas en Argentina también son fácilmente reconocibles. 
Prebisch, luego de la experiencia de la Gran Depresión, era consciente de los graves problemas que podía traer la deflación. Ésta era la diferencia más importante con el proyecto británico. Aprovechando la revaluación del oro en la Caja de Conversión, se creaba el BCRA, se resolvían los problemas de liquidez del sistema y se creaba el гмів para lidiar con la insolvencia del sistema bancario. El BCRA contaba con todos los instrumentos para llevar adelante una política anticíclica, de acuerdo a la visión del ciclo económico argentino que tenía Prebisch. Los instrumentos a disposición del banco central permitían intervenir sobre todos los mecanismos de transmisión que actuaban en las fases ascendentes y descendentes del ciclo.

Aunque muchas veces se presenta el BCRA como una creación en base al proyecto de Niemeyer, el papel de Prebisch fue fundamental y su proyecto se diferencia del británico en tres aspectos fundamentales.

En primer lugar, en el proyecto de 1934, Prebisch concibió un sistema de control de cambios que podía proveer estabilidad sin las rigideces de la convertibilidad. Las diferencias con el proyecto de Niemeyer no estaban solamente en la oportunidad o no de adherir al patrón oro, sino en la forma que éste debía adoptar en el caso de ser implementado. Prebisch se aseguró que en el caso de que fuera implementado, fuese un patrón oro y no un patrón de cambio oro. Después de la crisis, Prebisch era perfectamente consciente de los riesgos que implicaba mantener reservas en divisas. Niemeyer, por el contrario, temía que un masivo retorno al patrón oro pudiese impedir a los países europeos acumular reservas suficientes. La constitución de un sterling bloc donde fuese implementado el patrón de cambio oro era una de las prioridades británicas.

En segundo lugar, había desacuerdos en cuanto a la composición y los instrumentos del BCRA. Niemeyer temía la participación estatal porque estaba obsesionado con la inflación causada por el déficit fiscal excesivo. Prebisch, si bien estaba a favor de una participación limitada del Estado, lo incluyó como accionista del banco, con una participación de $50 \%$ y le acreditó los beneficios de la revaluación del oro. 
Con respecto a los instrumentos, Niemeyer pensaba que el banco debía intervenir en el mercado sólo mediante la tasa de redescuento. Prebisch, por el contrario, tenía en claro que había que esterilizar las fluctuaciones del capital externo. Para lograr este propósito, el redescuento no era suficienteł era necesario permitir al banco realizar operaciones de mercado abierto. Además, en el caso de un aumento de precios que incentivase las importaciones, el banco contaba con la posibilidad de implementar controles cambiarios para controlarlas. Finalmente, para evitar que se perpetuase la costumbre de los bancos de presentar balances falsos y su comportamiento irresponsable, la supervisión de los bancos estaba incluida entre las funciones del banco central. Con todos estos instrumentos, todas las áreas relevantes estaban cubiertas.

Finalmente, la tercera importante contribución de Prebisch fue la revaluación del oro y su uso por el gobierno. Niemeyer, que pensaba en un futuro retorno a la convertibilidad, veía a la revaluación como una forma de fijar el precio del oro prematuramente, a un precio que probablemente no sería el definitivo. El beneficio de revaluación era visto como un beneficio temporal que engendraría un proceso inflacionario. Estos temores se veían acentuados por la participación estatal en el гмів. Niemeyer no había pensado en ninguna solución a la insolvencia de los bancos, ni pensaba que el sistema bancario necesitara liquidez adicional.

El temor a la inflación y a la participación estatal son una constante en el proyecto Niemeyer, que consideraba que el equilibrio fiscal era necesario para mantener el valor de la moneda establecido por ley. Asimismo, intentaba limitar al mínimo las ocasiones que pudiesen dar lugar a decisiones discrecionales por parte de las autoridades del banco central.

Para Niemeyer el proyecto de Prebisch reflejaba: «the general abandonment during the crisis of all decent principles of Central Banking and Public Finance» (Niemeyer, «Note on Mr. Connolly's Comments», 2nd June 1933).

Prebisch, a pesar de que era conservador en cuanto a la participación estatal, reconocía que el Estado debía tener un papel más importante. Como gran conocedor de la economía argentina, reconocía la necesidad de dejar un 
margen considerable de acción a las autoridades monetarias, evitando imponer reglas demasiado estrictas. Además, conocía bien los riesgos de la deflación, que lo preocupaban más que los riesgos de inflación. Sin embargo, en sus conversaciones en el Banco de México en 1944, Prebisch diría que su proyecto de 1934 era ortodoxo, dado que tenía como objetivo la estabilidad del valor de la moneda y no la estabilidad de la actividad económica interna. El proyecto de política monetaria presentado en el Banco de México en 1944 estaba dirigido a este segundo objetivo.

\section{RefERENCIAS}

Banco de la Nación Argentina (1928-1934), Revista Económica del BNA, desde el núm. 1 de 1928 hasta núm. 9-12 de 1934.

Ford, A. G. (1962), The Gold Standard 1880-1914: Britain and Argentina, Oxford, Clarendon Press.

Furtado, C. (1988), La fantasía organizada, Buenos Aires, eudeba.

Helleiner, E. (2009), «Central Bankers as Good Neighbours: us money doctors in Latin America during the 1940s», Financial History Review, 16 (1), pp. 5-25.

Hirschman, A. (1961), «Ideologies of Economic Development in Latin America», en A. Hirschman, Latin American Issues: Essays and Comments, New York, Twentieth Century Fund.

Magariños, M. (1991), Diálogos con Raúl Prebisch, México, Bancomex, Fondo de Cultura Económica.

Niemeyer, O. [1933a], «Informe de Sir Otto Niemeyer», Revista Económica del BNA, vol. 7, núm. 5-8, 1934, pp.131-143.

(1934). «Algunas cuestiones suscitadas por Sir Otto Niemeyer», Revista Económica del BNA, vol. 7, núm. 5-8, 1934, p. 106.

Nurkse, R. y W. Adams Brown (1944), International Currency Experience: Lessons of the Inter-War Period, Geneva, League of Nations. 
O’Connell, A. (1984), «La Argentina en la depresión: los problemas de una economía abierta», Desarrollo Económico, núm. 23, pp. 479-514.

Pazos, F. (1988), «Raúl Prebisch, banquero central», Revista de la CEPAL, núm. 34, pp. 189-204.

Pérez Caldentey, E. y M. Vernengo, «Retrato de un joven economista: la evolución de las opiniones de Raúl Prebisch sobre el ciclo económico y el dinero, 1919-1949», Revista de la CEPAL, núm. 106, pp. 7-22.

Prebisch, R. [1921-1922], «Anotaciones sobre nuestro medio circulante. A propósito del último libro del doctor Norberto Piñero», Raúl Prebisch: Obras 1919-1948 (1991), vol. 1, Buenos Aires, Fundación Raúl Prebisch, pp. 93-175.

[1931], «Redescuento», Raúl Prebischઃ Obras 1919-1948 (1991), vol. 2, Buenos Aires, Fundación Raúl Prebisch, pp. 1-3.

[1932], «La acción de emergencia en el problema monetario», en Raúl Prebisch: Obras 1919-1948 (1991), vol. 2, Buenos Aires, Fundación Raúl Prebisch, pp. 42-67.

(1934), «Exposición de motivos de los proyectos sobre bancos y moneda», Revista Económica del BNA, vol. 7, núm. 5-8, pp. 61-96.

[1944], «El control de cambios en la república Argentina», en Raúl Prebisch: Obras 1919-1948 (1991), vol. 3, Buenos Aires, Fundación Raúl Prebisch, pp. 10-87.

[1949], «El desarrollo económico de la América Latina y algunos de sus principales problemas», en Raúl Prebisch: Obras 1919-1948 (1993), vol. 4, Buenos Aires, Fundación Raúl Prebisch, pp. 490-551.

Sayers, R. S. (1976), The Bank of England 1891-1944, 3 volúmenes, Cambridge, Cambridge University Press.

Sember, F. (2010). «The origins and evolution of the monetary thought of Raúl Prebisch», tesis doctoral, Università di Macerata y Université de Paris 1 Panthéon-Sorbonne.

Triffin, R. (1966), The World Money Maze: National Currencies in International Payments, New Haven and London, Yale University Press. 
Tugan-Baranowsky, M. (1913), Les crises industrielles en Angleterre, Paris, M. Giard \& E. Brière.

Fuentes inéditas

Niemeyer to Meynell (26 $6^{\text {th }}$ may 1933), Archives of the Bank of England, OV102/203.

Niemeyer, «Note on Mr. Connolly's Comments», $2^{\text {nd }}$ June 1933, Archives of the Bank of England, OV102/203

Meynell to Peacock $\left(10^{\text {th }}\right.$ december 1934), Archives of the Bank of England, ov102/203. 